\title{
Retinitis Pigmentosa: Pathophysiology and its Management
}

\section{Muhammad Imran Qadir*, Shahida}

Institute of Molecular Biology and Biotechnology, Bahauddin Zakariya University, Multan, Pakistan

*Corresponding Author: Muhammad Imran Qadir, Institute of Molecular Biology and Biotechnology, Bahauddin Zakariya University, Multan, Pakistan, Tel: +92-301-6515613; Fax: +92-061-9210098; E-mail: mrimranqadir@hotmail.com

Received: 18 May 2018; Accepted: 28 June 2018; Published: 06 July 2018

\begin{abstract}
Retinitis pigmentosa (RP) is a degenerative disease of retina which involves the pigment deposition in periphery of retina. In RP, there is degeneration of the rods and cones. So, RP is called as cone and rod dystrophy. Occurrence of RP is 1 in 4000 in human population. Major forms in which RP exist are simple, syndromic, non-syndromic. Photophobia, patchy loss of peripheral vision, tunnel vision, and color perception impairment are the symptoms of Retinitis pigmentosa. Electroretinogram (ERG), Electro-oculogram (EOG) are the diagnostic test utilized to diagnose RP. Different treatments are utilized to treat retinitis pigmentosa.
\end{abstract}

Keywords: Retinitis pigmentosa; Electroretinogram; Electro-oculogram; RNA interference; Gene therapy

\section{Introduction}

Eye is a complex organ of a human body. It makes human able to visualize different things with different colors. Eye is composed of different layers i.e. cornea, lens, retina (it further contain rods and cones), optic nerve. The conversion of light signal to the visual signals, which are then transmitted to the brain is actually done by the retina. Retina responds to light through sensory neuron and neural circuits. Retina layer contains two types of photoreceptor, 120 million rod and 1 million cones. Rod and cone are distinguished by their structure, types of photo-pigments, retinal distribution and pattern of synaptic connection as shown in Figure 1. These properties show that cone and rod system is specialized for various aspects of vision. Rods have discs, similar to stacked coins. Rods function best in low light and cones in bright light of visible spectrum. Rods are more sensitive as compared to cones and they function in dim light. Fovea is a region present in the central segment of retina called as macula. Fovea contains great number of cones photoreceptor density. $75 \%$ of the incident light reaches the fovea, it actually provokes greater degree of visual acuity. Inner region of the fovea contains 30 cones and it absorb one quarter of the 
photon. Rest of the photon is received by the cones of outer region of fovea. The probability of photon absorbed by a cone depends upon the following factors:

1. The direction of photon (most efficient direction of photon is along the axis of cone).

2. Frequency of approaching photon.

3. Types of cones (L, M, S type) [1].
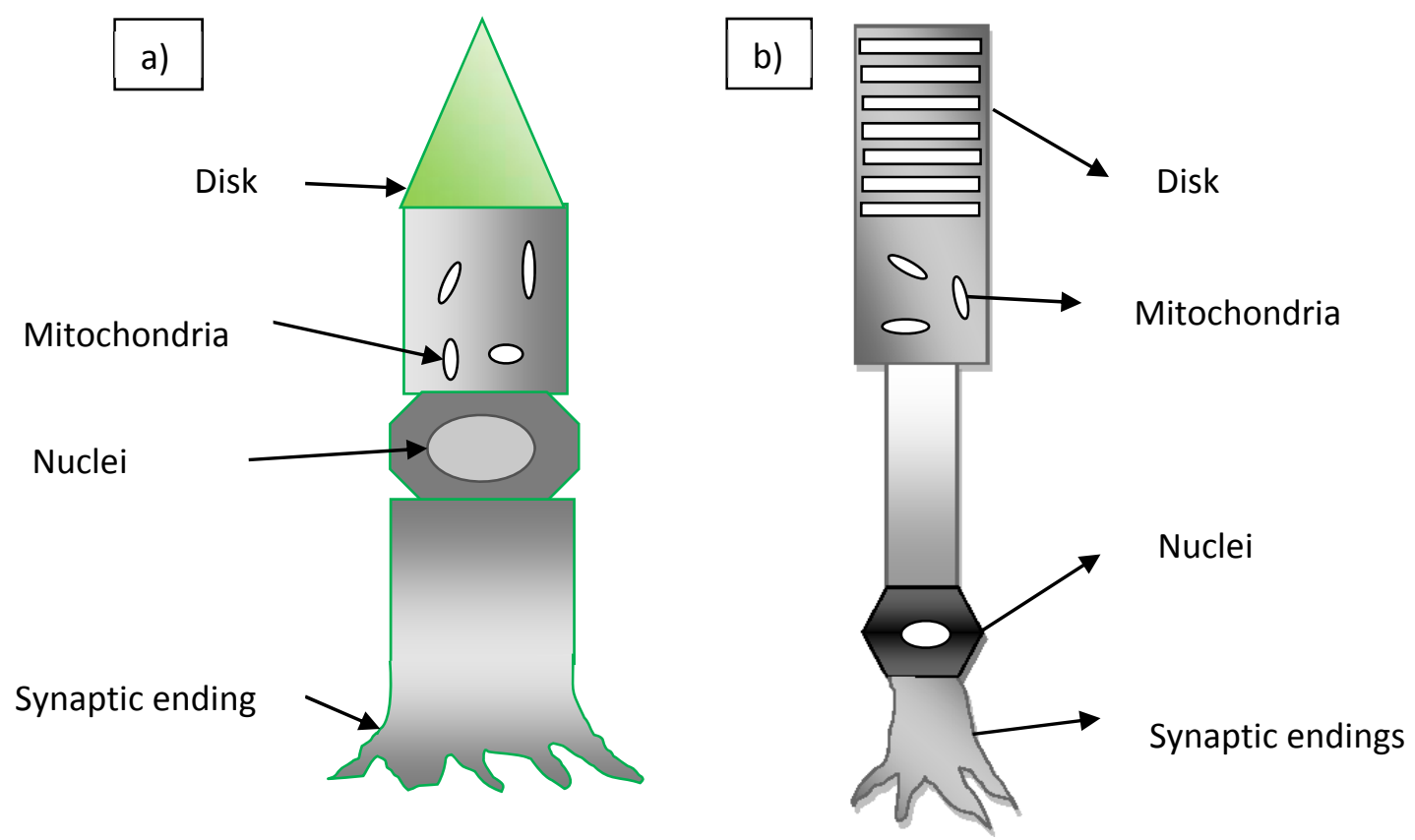

Figure 1: a) Eye cone structure, b) Eye rod structure.

Retinitis pigmentosa (RP) is a degenerative disease of retina followed by pigment deposition in periphery of retina as shown in Figure 2. In RP there is a major degeneration of the photoreceptor rods, with minor degeneration of the cones. So RP is known as cone and rod dystrophy. RP starts from mid periphery and then moves toward macula and fovea. Prevalence of RP is 1 in 4000 in human population. Symptoms of RP appears in early teenage but it reaches to severe visual impairment at the age of 40 to 50 year. RP exist in different forms, simple, syndromic, non syndromic. In syndromic form pleiotropic effect is dominant. Nonsyndromic cases include; roughly adRP are 30\%, autosomal recessive RP are 20\%, X-linked RP are 15\%, and 5\% are early-onset forms of RP that are normally diagnosed as recessive LCA. Recessive RP is caused by the mutation in 25 genes. Recessive LCA is caused by the mutation in 13 genes. Dominant LCA is caused by the mutation in 2 genes. X-linked RP is caused by the mutation of 6 genes. 20- $30 \%$ of the patients of RP are having syndromic RP and associated with non-ocular disease [1, 2]. 
Clinical findings include: Pigment clumping or bone spicule pigmentation, waxy pallor of optic nerve, retinal arteriolar narrowing, formation of atrophy of the RPE, epiretinal membrane and choriocapillaris, posterior subcapsular cataract, epiretinal membrane formation, and cystoid macular edema (CME).[1].

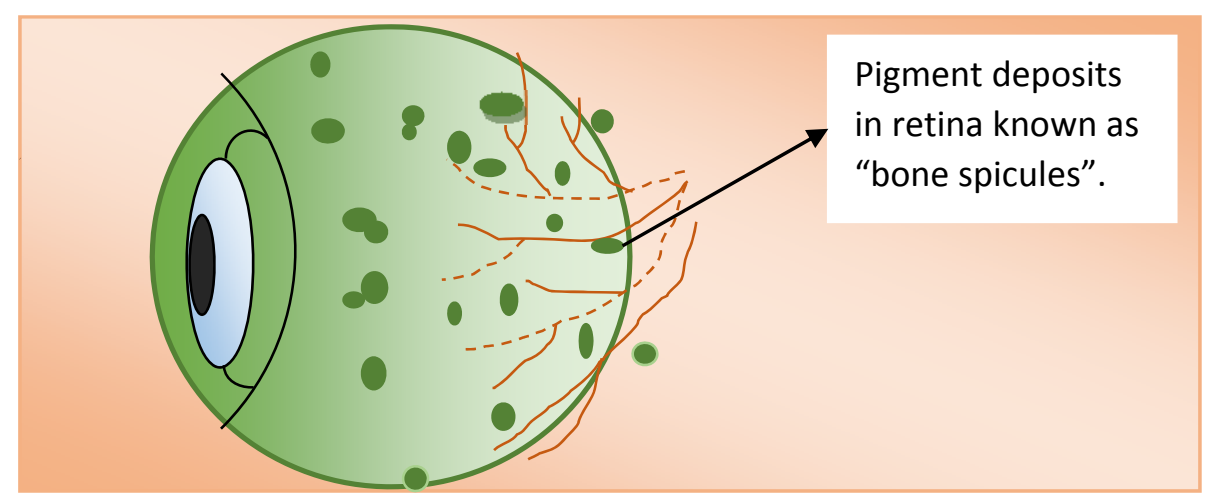

Figure 2: Eye affected by retinitis pigmentosa.

\section{Symptoms}

The symptoms of retinitis pigmentosa are night blindness in early stage, photophobia, patchy loss of peripheral vision, tunnel vision, and color perception impairment [2].

\section{Epidemiology}

In early stage of the RP night blindness is the main symptoms that might appear in first year of life or in the second decade or even late. Patients usually ignore mild night blindness but they may have peripheral visual field defects dim light. Diagnosis of RP becomes more difficult when the patient do not have any familial history. In the mid stage of the disease night blindness is obvious. Patient in the day light also faces the loss in the peripheral visual field. Person suffering from the RP in the mid stage also become photophobic to diffuse light. The end stage of the $\mathrm{RP}$ is characterized by peripheral vision loss (classical tunnel vision), intense photophobia, and few degree of vision [2].

\section{Mode of Inheritance}

Inheritance of Retinitis pigmentosa from parents to their children is observed. Autosomal dominant are mildest forms starting after the age of 50 in some cases. During first decade of life autosomal recessive form starts. X-linked forms also start in early stage of RP and associate with myopia.

\section{Syndromes Associated with Retinitis Pigmentosa}

There are different syndromes associated with RP. Some of these syndromes are 
Fortune J Health Sci 2018; 1 (1): 019-025

DOI: 10.26502/fjhs003

\subsection{Usher syndrome}

It is a syndrome which associates RP with neurosensory deafness. 14\% of RP cases suffer from usher syndrome. At least mutation in 11 genes lead to usher syndrome [3].

\subsection{Bardet Biedl Syndrome}

Usher syndrome is more frequent than Bardet Biedl syndrome [4].

\subsection{Senior Loken Syndrome (SLK)}

It usually associated RP with nephronophtisis.4 genes involve in the encoding of nephrocystins are linked to this disease.

\subsection{Cohen syndrome}

It associates RP to facial dysmorphism, mental retardation, short stature, neutropenia and long narrow hands. The electroretinogram, the measure of retinal function describes the function of photoreceptors of eye. 45 genes responsible for RP in 60\% patients suffering and the defect of remaining ones are unidentified [4].

\section{Diagnostic Tests}

Different laboratory test has been designed to test the RP conditions:

> Neoplasm is associated to study antiretinal antibodies (particularly anti-recoverin antibodies), particularly in cancer-associated retinopathy (CAR) or in severe RP.

$>$ Electroretinogram (ERG): For RP it is most critical diagnostic test.

$>$ Electro-oculogram (EOG): Not helpful in diagnosing RP, but essential to find changes in macular, normal findings of ERG and unusual EOG findings suggest Best vitelliform macular dystrophy.

$>$ Formal visual field testing: helpful to measure continuous follow-up care of RP patients.

$>$ Color testing: Commonly use to check, mild blue-yellow axis color defects, usually RP patients do not face difficulty with color perception.

> Dark adaptation study: Disproportionately decrease contrast sensitivity relative to visual acuity in RP; bright-light sensitivity.

\section{Treatments of Retinitis Pigmentosa}

\subsection{Gene therapy}

Gene therapy is a technique in which genes are utilized to treat or prevent disease. This is done by replacing the defective gene, by in activating of nock out of the defective gene, by inserting the desired gene. This technique plays vital role in treating variety of inherited human diseases. Genetic modification of mutant ocular cell is done by the ocular gene therapy. RP can also efficiently treated by the ocular gene therapy. After finding the genetic etiology photoreceptors or retinal pigment epithelium cells can be targeted by subretinal injection. Strategies of gene therapy 
Fortune J Health Sci 2018; 1 (1): 019-025

DOI: $10.26502 / f j h s 003$

may vary depending upon the inheritance of RP or the type of mutation. In USA, UK, Italy ocular gene therapy trial was done on the patients having mutation in RPE65 gene and encouraging results were obtained [5].

\subsection{Neurotrophic factors}

Protection of photoreceptors from degeneration can be done by several neurotropic factors including ciliary neurotrophic factor. Test of CNTF on animals models suffering from RP show delay in photoreceptor degeneration, as thick layer was found in treated animals which was seem to protect photoreceptors. Enhancement in scotopic and photopic responses by using CNTF can be checked by the electrophysiological recordings are performed.

\subsection{Stem cells}

It is a technique in which embryonic stem cells are used to produce degenerative cells. Enzmann and colleague use the stem cells, their elasticity and property to produce particular cells and ability for self renewal. Further Lund and his coworkers used RP embryonic cells (RPE) of photoreceptors obtained embryonic stem cells of human. He then implanted RPE cells in the rats suffering from retinal degenerative disease. Results obtain show $100 \%$ improvement in RP as compared to the untreated rats [5].

\subsection{Light protection}

Patients suffering from RP should protect their eyes from ultraviolent rays and visible wavelength up to 527nm. Protection of eyes can be done by using amber spectacles outdoor, which actually blocks the ultraviolent rays and light of approximately of wavelength $550 \mathrm{~nm}$ [5].

\subsection{Vitamin therapy}

Vitamin A shows tropic and antioxidant effect so they may protect photoreceptors. Supplements of vitamin A ma reduce the degeneration of photoreceptors. Scientists found that in addition to vitamin A, DHA supplement at $1200 \mathrm{mg} /$ day is given to the RP patients as a result improvement was observed [6].

\subsection{RNA interference}

RNA interference (RNAi) involve sequence-specific, post-transcriptionl silencing of gene initiated by double stranded RNA (dsRNA) that is complementary in sequence to the mRNA of the targeted gene. The principle of RNAi is that an enzyme called as dicer, which cuts the long dsRNA into short fragments. These small fragments are called as small interfering RNAs (siRNA). These 21-24 nucleotide duplexes enter in the so-called RNA-induced silencing complex (RISC) where the duplex is unwound. Argonaute protein actually degrades the one strand of dssRNA, leaving the remaining strand for binding to targeted mRNA sequence according to base pairing rule. A binds to $\mathrm{T}$ and $\mathrm{G}$ to $\mathrm{C}$. Once siRNA binds to the targeted mRNA, argonaute protein then degrade the target RNA as shown in Figure 3. Gene that encourage oxidative damage or apoptosis in retinal cells has been identified by RNAi [5]. 


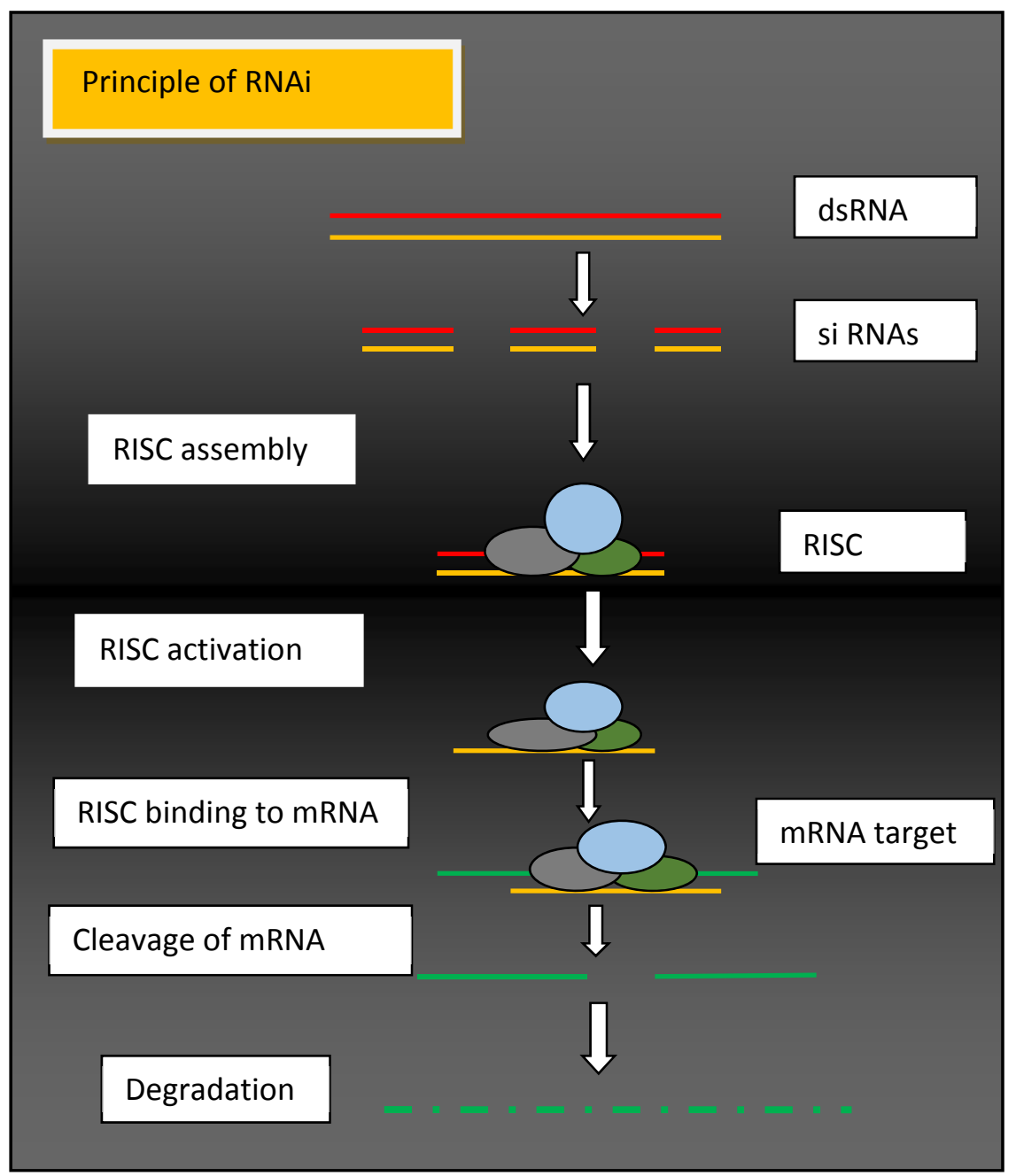

Figure 3: Principle of RNAi, dsRNA is converted into siRNA, after this RISC complex is formed, which actually degrade the one strand of dsRNA, then ssRNA binds to the target mRNA and cleave it.

\section{Conclusion}

Retina pigmentosa is an eye disorder, Different treatments strategies are being adopted to cure or to minimize the effect of disease.

\section{References}

1. Mustafi D, Engel AH, Palczewski K. Structure of cone photoreceptors. Progress in Retinal and Eye Research 28 (2009): 289-302.

2. Hamel C. Retinitis pigmentosa. Orphanet Journal of Rare Diseases 1 (2006): 40.

3. Roux AF. Données moléculaires du syndrome de Usher. Journal Francais d'Ophtalmologie 28 (2005): 9397. 
Fortune J Health Sci 2018; 1 (1): 019-025

DOI: $10.26502 / f j h s 003$

4. Beales P, Elcioglu N, Woolf A, et al. New criteria for improved diagnosis of Bardet-Biedl syndrome: results of a population survey. Journal of Medical Genetics 36 (1999): 437-446.

5. Musarella MA, MacDonald IM. Current concepts in the treatment of retinitis pigmentosa. Journal of Ophthalmology (2011).

6. Berson EL, Rosner B, Sandberg MA, et al. Further Evaluation of Docosahexaenoic Acid in Patients With RetinitisPigmentosa Receiving Vitamin A Treatment: Subgroup Analyses. Archives of Ophthalmology 122 (2004): 1306-1314.

Citation: Muhammad Imran Qadir, Shahida. Retinitis Pigmentosa: Pathophysiology and its Management. Fortune Journal of Health Sciences 1 (2018): 019-025.

(C) (P) This article is an open access article distributed under the terms and conditions of the Creative Commons Attribution (CC-BY) license 4.0 